\title{
Bemerkungen zu H. Fischers Entgegnung und seiner Beurteilung der spektroskopischen Methode. \\ Von
}

O. Schumm.

(Der Redaktion zugegangen am 21. Juli 1916.)

In H. Fischers Abhandlung (Über das Urinporphyrin» ') findet sich (S. 37) der Ausspruch «Spektroskopisch stellte Günther die absolute Übereinstimmung des im Urin enthaltenen Porphyrins mit Hämatoporphyrin fest und neuerdings wieder $\mathrm{O}$. Schumm in Hamburgs. Diesen Ausspruch habe ich als estreng genommen nicht richtig* bezeichnet. ${ }^{2}$ ) H. Fischer beanstandet nun, ${ }^{3}$ ) daß ich in meiner Begründung das spektroskopische Verhalten des Harns mit dem einer Lösung des Hämatoporphyrins in Soda und mit ammoniakalischen Lösungen von Urinporphyrinpräparaten verglichen habe. Daß̉ die so festgestellten Abweichungen keine vollkommen sichere Beurteilung gestatten, ist mir natïrlich bekannt gewesen, denn ich habe dazu selbst bemerkt, daß es nicht sicher entschieden sei, ob dieser Unterschied auf den EinfluB von Harnbestandteilen bezw. die Reaktion zurückgeführt werden könne, ${ }^{4}$ ) daß man diese Erscheinung aber nicht übergehen könne, da sie nicht vereinzelt sei. Der Unterschied in der Lage des I. Absorptionsstreifens (im Rot) der Porphyrinharne und des Hämatoporphyrins von Nencki wird, wie ich schon damals gezeigt habe, noch größer, wenn man den Harn stark alkalisiert. ${ }^{5}$ ) Anderseits habe ich schon früher, in meiner Abhandlnng über Hämatoporphyrin und

1) Diese Zeitschrift, Bd. 95, S. 37.

^) 0.S chu m m, Über das «Hämatoporphyrin ` aus Harn und Knochen, Diese Zeitschr., Bd. 96, S. 191, 1915.

3) H. Fischer, Über die Giftigkeit, die sensibilisierende Wirkung und das spektroskopische Verhalten der natürlichen Porphyrine. Abbau des Urinporphyrins zum Kotporphyrin. Diese Zeitschr., Bd. 97, S. 114.

4) E. Roedelins und 0. Schumm, Über Hämatoporphyrinogenausscheidung im Harn. Zeitschr. f. Urologische Chirurgie, Bd. III, H. 1/2, Zweiter Teil: O. Schumm, Die Farbstoffe des Harns, S. 125.

5) O.Sch u mm, Über Vorkommen und Nachweis einiger pathologisch wichtiger Abbauprodukte des Blutfarbstoffs. Festschrift des Eppendorfer Krankenkauses, S. 198 (auch Anm. 1). Verlag von L. Voss, Leipzig und Hamburg, 1914.

Hoppe-Seyler's Zeitschrift f. physiol. Chemie. XCVIII. 
Nesoporphyrin ') angeben können, daß man bei Lösungen von Nenckis Hämatoporphyrin den Gehalt an Alkali in ziemlich weiten Grenzen ändern kann, ohne dab eine bedeutende Änderung im Ort der Streifen auftritt, somit auch der I. Streifen (im Rot) seine gegenüber dem I. Streifen des alkalisierten Porphyrinharns stark abweichende Lage beibehält. Es ist viclleicht eine etwas weitgehende Vorsicht von mir gewesen, daß ich aus dieser von mir betonten und berbachteten Abweichung nicht ohne weiteres den Schluß gezogen habe, daß das Harnhämatoporphyrin in seiner chemischen Konstitution von Nenckis Hämatoporphyrin irgendwie verschieden sei. Meine Untersuchung an $G$ ünther-Fischers $F$ all beschränkte sich aber auf eine Einzelportion eines nicht vollkommen frischen, sondern von Bonn eingesandten Harns. Ich begnügte mich deshalb damit, die bei den Spektren der alkalischen Flüssigkeiten beobachteten Abweichungen zu erwähnen. An anderer Stelle habe ich dann noch betont, ${ }^{2}$ ) daB man sie nicht übergehen könne. H. Fischers Angabe, daB ich spektroskopisch die absolute Übereinstimmang des im Urin enthaltenen Porphyrins mit Hämatoporphyrin festgestellt habe, ist demnach nicht richtig. Die von mir gefundene Abweichung in den Spektren der salzsauren Lösung des roben Harnbämatoporphyrins (Kalifällung des Harnes) und des Hämatoporphyrins von Nencki ist allerdings gering (rund $1 \mu \mu$ ). Meine Beobachtung ist aber richtig gewesen. da die Absorptionsstreifen des reinen Harnhämatoporphyrins in $25 \%$ iger Salzsäure, wie sich jetzt bestimmt behaupten läßt, ${ }^{3}$ ) tatsächlich weiter nach Rot liegen als beim Hämatoporphyrin von Nencki. H. Fischers Ausspruch, daß Unterschiede von $1 \mu \mu$ innerhalb der Fehlergrenze liegen, kann ich nur für solche Fälle zustimmen, in denen es sich um weniger genau bestimmbare Streifen handelt. Bei den Spektren der Porphyrine des Harns, sowie von Nenckis Hämatoporphyrin und Mesoporphyrin in wässeriger Salzsäure lassen sich aber wenigstens der erste und dritte Streifen sehr genau bestimmen, wenn man vorschriftsmäßig die Okularmarke auf die dunkelste Stelle oder (bei breiteren symmetrischen Streifen) auf die Mitte oder (bei Streifen mit deutlichem schmalen Minimum, zum Beispiel bei Fischers Kotporphyrin) auf das Minimum einstellt, wobei es nicht, wie H. Fischer meint, besonders darauf ankommt, daß man den prozentualen, Gehalt der Lösung an Farbstoff kennt, sondern darauf, daß die Lösung dén jeweils zu messenden Streifen in der für die genaueste Messung richtigen Stärke zeigt. Da nun die einzelnen Streifen des Absorptionsbildes einer salzsauren Porphyrinlösung sehr verschieden stark sind, so folgt daraus, daß man die Ortsbestimmung der einzelnen

1) O.Schumm, Untersuchungen über die Absorptionserscheinungen des Hämatoporphyrins und Mesoporphyrins im Gitterspektrum. Diese Zeitschrift, Bd. 90, S. 12.

2) E. Roedelins und 0. Schumm, l. c., S. 125.

3) Vergl. meine nächste Abhandlung in dieser Zeitschrift. 
Streifen an Lösungen von verschiedenem Farbstoffgehalt ausführen muß! Genaueres darüber habe ich $u$. a. auch in dieser Zeitschrift in meiner Abhandlung :Über die Messung und Bestimmung der Absorptionsspektra», Bd. 66, S. 298 angegeben. Ein Beispiel für die bei den spektrometrischen Bestimmungen erzielbare Genauigkeit geben die Werte, die von For m a n e ck und von mir für den gut bestimmbaren ersten Streifen des 0xyhämoglobins gefunden sind (vgl. diese Zeitschrift, Bd. 83, S. 23). ${ }^{\text {) }}$

H. Fischers Ansicht, daB H. Günther spektroskopisch die absolute Übereinstimmung des im Urin enthaltenen Porphyrins mit Hämatoporphyrin festgestellt habe, ist ebenfalls irrtümlich. Die von $H$. Günther angegebenen Zahlen lassen sich freilich nur zum Teil richtig beurteilen, weil mehrfach nicht genau angegeben worden ist, wieviel Salzsäure das angewandte Lösungsmittel enthielt. Auf S. 136 seiner Abhandlung schreibt Günther, daß sich bei der Darstellung nach Saillet ein Teil des Porphyrins nicht mit $5 \%$ iger $\mathrm{HCl}$, wohl aber mit $25 \%$ iger $\mathrm{HCl}$ habe extrahieren lassen; die Lösung habe folgende Bänder gegeben: 596-589, 557-542. Daraus würde sich der Ort des ersten Streifens zu etwa 592,5, der des zweiten zu etwa 549,5 ergeben. Diese Zablen stimmen weder zu Nenckis Hämatoporphyrin noch zu dem Harnhämatoporphyrin (Fischers Urinporphyrin), wohl aber zu dem von Fischer auch im Harn dieses Patienten gefundenen Kotporphyrin! Unbestreitbar sind auch die bedeutenden Abweichungen gegenüber Hämatoporphyrin Nencki bei den ammoniakalischen Lösungen des von $H$. Günther aus Petrys Harn abgeschiedenen Farbstoffs. Für die ammoniakalischen Lösungen nach Saillets Verfahren gewonnener Präparate gibt G.ünther an: a) S. 136, Zeile 11/12: Zablen, aus denen sich der Ort des I. Streifens, im Rot, zu ungefähr 608 berechnet, ein Wert, der nicht annähernd für Nenckis Hämatoporphyrin, auch nicht für Kotporphyrin, wohl aber, wie man jetzt weiß, annäherd zu Fischers Urinporphyrin paßt; b) auf S. 136, Zeile 18: Zahlen, aus denen sich der I. Streifen zu ungefähr 614,5 berechnet; c) Zeile 23, zu 611; d) S. 137, Zeile 10, zu 607,5.

H. Fischer hat aus der Art, wie ich die Bezeichnung esaures Hämatoporphyrinspektrum> gelegentlich angewandt habe, geschlossen, daß ich Harnhämatoporphyrin und Nenckis Hämatoporphyrin für chemisch identische Stoffe gehalten habe. Der Ausdruck ssaures Hämatoporphyrinspektrum> ist für ein solches Absorptionsbild, das an verschiedenen bis-

1) Wie ich bei dieser Gelegenheit hervorheben möchte, habe ich bei der Ortsbestimmung der Absorptionsstreifen durch okulare Messungen die größte Genauigkeit erzielt, wenn ich mich eines Spektrometers bediente, das mit einem vertikalen Spalt ausgestattet war, während für die Bestimmung des Intensitätsverhältnisses durch gleichzeitige Beobachtung zweier Absorptionsspektra auch nach meinen Erfahrungen die Apparate mit horizontalem Spalt als geeigneter gelten dürfen. 
lang sogenannten sHämatoporphyrinen, in mineralsaurer Lösung beobachtet wird, gebräuchlich; doch ist obne weiteres zuzageben, daß diese übliche freie Anwendung des genannten Ausdrucks zu Mißverständnissen fähren kann. Leider ist in dem klinischen Teil unserer Abhandlung über einen Fall von ‘Hämatoporphyrinogenuries unbekannter Ätiologie (Zeitschr. f. Urologische Chirurgie III. S. 112) an einer Stelle ausgesprochen, daß anfangs die Ausscheidung von echtem Hämatoporphyrin im Sinne Nenckis, später dagegen die Porphyrinogenausscheidung überwogen habe. Dieser Ausspruch sollte nur eine Gegenüberstellung der Begriffe Hămatoporphyrinfarbstoff und Porphyrinogen geben. Der Zusatz sim Sinne Nenckis, hätte unterbleiben müssen, weil er eine nicht beabsichtigte Auslegung des ganzen Ausspruchs nahelegte. In dem von mir verfaßten $z$ weiten, dem chernischen Teil derselben Abhandlung habe ich die in der Überschrift des vorletzten Abschnitts aufgeworfene Frage sIst das Porphyrin des Harns mit einem der von Nencki und Zaleski aus Blutfarbstoff dargestellten Porphyrine identisch? 2 mangels genügend sicherer Unterlagen nicht bestimmt beantwortet, auch gerade in dieser Schlußbesprechung (S. 125) die Abweichungen im spektralen Verhalten zwischen Harnhämatoporphyrin und Nenckis Hämatoporphyrin hervorgehoben.

Auf Seite 113 seiner Abhandlung nimmt $\mathrm{H}$. Fischer Bezug auf die Zitate aus meiner anderen, zusammenfassenden Abhandlung, die zum Ausdruck bringen, daß die Übereinstimmung der Spektren de3 Harnporphyrins mit Nenckis Hämatoporphyrin keine vollständige, sondern nur eine angenäherte ist, und schreibt: ${ }^{1}$ ) «Ich habe diese Einschränkungen als eine Captatio benevolentiae mir gegenüber aufgefaßt, da die aus der angezogenen Arbeit von Schumm angeführten Zitate S. 191 eingeleitet werden: sImmerhin besteht der von $\mathrm{H}$. Fischer und Meyer-Betz erhobene Einwand in gewissem Umfange zurecht: cIn einer Anzahl von Fällen genügen die Angaben der Untersucher nämlich nicht, um zu beweisen, daß Hämatoporphyrin und nicht etwa Mesoporphyrin oder ein anderes Porphyrinvorgelegen habe.s Der Ausspruch Fischers ist mir nicht verständlich und seine Angabe über diesen meine Zitate einleitenden Satz unrichtig; er steht zwar auf Seite 191, danach folgt aber eine mehrere Seiten lange Auseinandersetzung über einige Porphyrine, unter anderem eine zwei Seiten lange Schilderung ihres Verhaltens bei Arnolds Bromreaktion und erst auf Seite 196 folgen die Zitate, die durch folgenden, auch dem Sinne nach ganz anderen Satz eingeleitet werden: \& Unter Verwertung dieser und der an anderer Stelle ausführlich mitgeteilten Befunde läßt sich bezüglich eines Teils der sorgfältiger untersuchten Fälle von sogenannter Hämatoporphyrinurie entscheiden, ob der beobachtete Farbstoff in seinem spektralanalytischen Verhalten

1) H. Fischer, 1. c., Diese Zeitschr., Bd. 97, S. 114, 1916. 
die Merkmale von Nenckis Hämatoporphyrin oder Mesoporphyrin gezeigt hat. Ich greife an dieser Stelle nur einige von H. Günther angeführte Fälle von (kongenitaler) Hämatoporphyrie, die von 0 . Hammarsten untersuchten Fälle von sogenannter Sulfonalhämatoporphyrinurie und zwei der hier beobachteten Fälle als Beispiel heraus.> Es folgen 8 Zitate und unmittelbar darauf die die Besprechung dieser Frage abschließenden Sätze: <Ob bei den sonst noch beschriebenen Fällen sogenannter Hämatoporphyrinurie die Auffassung des Harnfarbstoffs als Hämatoporphyrin stets berechtigt war, möge einstweilen dahingestellt bleiben. Nach Entscheidung der Vorfrage, ob ein im Harn gefundenes Porphyrin in seinem spektralanalytischen Verhalten dem Hämatoporphyrin oder Mesoporphyrin am nächsten steht, bleibt noch die Frage offen, ob es zum Beispiel im ersten Falle mit dem Hämatoporphyrin <Nencki > in chemischer Hinsicht völlig identisch ist, oder ob es sich um ein etwas abweichend zusammengesetztes besonderes ‘ Harnhämatoporphyrin, handelt. Die Entscheidung dieser Frage ist wohl nur dann möglich, wenn es gelingt, größere Mengen Harnhämatoporphyrin zu krystallisieren und die gereinigten Krystalle einer eingehenden chemischen Analyse und auch der Prüfung auf eine etwaige photodynamische Wirkung zu unterwerfen. Dies hat sich bis jetzt noch in keinem Falle durchführen lassen.s Mit diesen Sätzen, die in meiner größeren, die eigenen Erfahrungen zusammenfassenden Abhandlung übor pathologisch wichtige Abbauprodukte des Blutfarbstoffs stehen, ist die Besprechung über das Harnhämatoporphyrin beschlossen.

Es ist ersichtlich, daß ich die Bezeichnung Hämatoporphyrin nicht lediglich für das Nenckische Präparat, sondern mehrfach als Gruppennamen ${ }^{1}$ ) gebraucht habe, demnach die Existenz mehrerer chemisch nahe verwandter \&Hämatoporphyrine> voraussetzte. Die chemische Identität von Harnhämatoporphyrin und Nenckis Hämatoporphyrin habe ich nicht behauptet, sondern als fraglich hingestellt.

Durch meine spektrometrischen Untersuchungen war übrigens für mehrere Formen von Hämatorphyrinurie (Sulfonalvergiftung, Hämatoporphyria congenita, ein Fall unbekannter Ätiologie) schon mit Sicherheit nachgewiesen, daß der vorwaltende Farbstoff dieser Harne nicht mit Mesoporphyrin identisch sei, eine Feststellung, die auf rein chemischem Wege für den Fall von Hämatoporphyria congenita von H. Fischer bestätigt ist. Weiterhin verdanken wir H. Fischer die sehr wich: tige Feststellung, daß bei diesem Falle der vorwaltende $\mathrm{Be}$ standteil des Harnfarbstoffs, Fischers "Urinporphyrin», in seiner elementaren Zusammensetzung und Atomgruppierung

1) Ebenso verfährt zum Beispiel auch $H$. Thierfelder, vergl. Hoppe-Seylers Handbuch der chemischen Analyse. 8. Auflage von H. Thierfelder, Berlin 1909, S. 364. 
vom Hämaphorphyrin Nencki beträchtlich abweicht, ihm in seiner pharmakologischen Wirkung aber ähnlich ist.

An mehreren Stellen seiner Arbeiten beschreibt H. Fischer, daB ihm spektroskopische Proben versagt hätten, und sucht zu zeigen, daß der Anwendung der Spektroskopie engere Grenzen gezogen werden müßten. Ich stimme dem voll und ganz zu, soweit es sich um Untersuchungen handelt, in denen die Methode in offenbar unzulänglicher Art, sei es an ungeeigneten Gegenständen oder mit ungeeigneten Apparaten angewandt worden ist. Auch bei Fällen, in denen die Möglichkeit besteht, durch Anwendung einer rein chemischen Methode mehr zu erreichen, ist dieser der Vorzug zu geben, vorausgesetzt, daß die äußeren Umstände ihre Anwendung nicht ausschließen. Es erscheint ferner nicht ratsam, in wissenschaftlichen Arbeiten bei der Beschreibung des spektralanalytischen Verhaltens eines Stoffes auch für genau bestimmbare Absorptionsstreifen Wellenlängenwerte zu veröffentlichen, die von den wahren Werten um mehrere $\mu \mu$ abweichen. Solche Angaben müssen unter Umständen zu irrigen Annahmen führen; sie sollten entweder mit dem Zusatz versehen sein, um wieviele $\mu \mu$ sie unrichtig sind oder mit dem Vermerk, daß es sich nur um ganz angenähert richtige Werte handelt.

H. Fischers Hinweis auf die aus den Grundlehren der Absorptionsspektralanalyse bekannte Tatsache, daB man mit Hilfe der spektroskopischen Beobachtung in Farbstoffgemischen die einem Einzelbestandteil eigentümliche Absorptionserscheinung in vielen Fällen nicht wahrnehmen kann, nimmt der spektroskopischen Methode nichts von der nur ihr eigenen Leistung, in vielen anderen Fällen die Anwesenheit selbst sehr geringer Mengen eines Farbstoffs auch in Farbstoffgemischen anzuzeigen. Eine schwierige, aber auch dankbare Aufgabe für den Spektroskopiker besteht gerade darin, auf Grund einer genauen Kenntnis des den einzelnen Farbstoffen eigentümlichen absorptiven Verhaltens auch die Mischspektren von Farbstoffgemengen richtig zu deuten. - Bei genügender Erfahrung wird man im allgemeinen schon vorher sagen können, in welchen Fällen eine chemischspektroskopische Untersuchung Erfolg verspricht. Da die Empfindlichkeit des spektroskopischen Nachweises für manche Farbstoffe eine außerordentlich hohe ist, so läßt sich durch diese Methode häufig noch weitgehende Aufklärung geben, wo an die Anwendung rein chemischer Methoden garnicht mehr zu denken ist. Schlagende Beweise hierfür bieten aus der Reihe wissenschaftlicher Feststellungen meine Mitteilungen in den Abhandlungen über das Hämatin im Blutserum ${ }^{2}{ }^{2}$ ) und über die pathologischen Farbstoffe des Blutes bei dem Falle von

1) 0. Schumm, Hämatinämie bei toxischem Blutkörperchenzerfall, Diese Zeitschrift, Bd. 80, 1912, S. 1.

2) Hämatin als pathologischer Bestandteil des Blutes, Diese Zeitschrift, Bd. 97, 1916, S. 32. 
llämatoporphyria congenita. ') Auf der anderen Seite gibt es eine große Anzabl ron Farbstoffen, die im sichtbaren Spektrum so schwacho odor so verschwommene Absorptionsorscheinungen geben, dab sie zu ihrem Nachweis oder ibrer Auffindung nur wenig odor garnicht geeignet sind. In solchen Fallen wird man natïrlich rein chemischen Methoden den Vorzug geben.

Zur erfolgreichen Anwendung der spektroskopischchemischen Methoden in der Biochemio sind vor allem böchst genaue in Wellenlängen ausgedrückte spektrometrische Grundwerte für Lösungen der reinen Farbstoffe in passend gewählten Lösungsmitteln notwendig. Es liegt sichor irn Intcresso der angewandten Biochemie, daB mit der Auffindung neuer biochemisch wichtiger Farbstoffe dio genaue Erforschung ihres absorptiven Verhaltens gleichen Schritt halte, besonders wenn es sich um Farbstoffe handell, die so a ugezeichnote Spektralerscheinungen geben wie dic Por. phyrino.

Auf H. Fischers Ausspruch, ") daß spoziell die chemischo Rrkenntnis der Natur des Harnporphyrins durch die Überschätzung der Wichtigkeit der spektroskopischen Bcobachtung aufgehalten worden sci, ist zu entgegnen, daß die Bahn für eine wirklich durchgreifonde rein chemische Bearbeitung des Harnporphyrins doch erst dadurch frei gemacht wurde, daß H. Fischer ausfindig machte, wie man den Farbstorf sicher in kiystallisierter Form bezw. in den Zustand zweifelsfreier Einheitlichkeit bringen konnte. Bis dahin war kein gangbarer Weg bekannt, auf dem man den Farbstoff in der für die erfolgreiche chernische Erforschung notwendigen sicheren Reinheit darstellen konnte! Zwar ist 0 . II a mmarsten ${ }^{3}$ ) bekanntich einige Male die Gewinnung eines krystallinischen Farbstofts aus Hümaloporphyrinharn gelungen, nicht jedoch in allen Fällen und nicht in einer für die genauero chemische Erforschung ausrcichendon Menge. Ich habo unter sinngomäßer Befolgung von 0 . Hammarstens Angaben in einem ältoren Falle von Hămatoporphyrinurie jenes kyrstallinische Produkt nicht erhalten können. $\mathrm{DaB}$ der von Nebeltha $\mathrm{u}$ eingeschlagene Weg, den Farbstoff durch Essigsăure abzuscheiden, nicht immer gangbar ist, habe ich schon friber angegeben. Neuerdings gibt auch $F$ ischer an, daB dieses Verfahren eine größero Farbstoffmenge voraussetze. ${ }^{4}$ ) Ohne Frago sind die Bedingungen

1) Vergl. die demnächst in dieser Zoitschrift orscheinende Abhandlung: Über die pathologischon Farbstoffe des Blutes bei Hämatoporphyria congenita.

2) H. Fischer, Beobachtungen am frischen Harn und Kol von Porphyrinpalienten, Diese Zeitschrift, Bd. 97, S. 166, 1916.

s) Olof Hammarsten, Uber Hămaloporphyrin im Harn. Skandinavisches Archiv für Physiologio, Bd. 3, 1892, S. 319-34:3.

4) 1. c. Bd. 97, S. 157. 
zu einer ziemlich vollständigen Ausfällung des Harnhämatoporphyrins aus dem Harn des Patienten Petry (bei dem Fischer die Reindarstellung gelang) besonders günstige. Auch habe ich in den letzten 10 Jahren, einschließlich des von mir im Jahre 1911 beschriebenen Falles, keinen Harn gesehen, der die Spektralerscheinung des Harmbämatoporphyrins in so reiner und ausgeprăgter Form gezeigt hätte wie die farbstoffreicheren Portionen bei diesem Falle. Die Ausfällang des Farbstoffes durch Essigsïure ist mir, seit der Kranke hier ist, fast ausnahmslos glatt gelungen. In einzelnen Portionen erfolgte die Ausscheidung des Farbstoffs nach Essigsäurezusatz verzögert. Jetzt, nachdem Fischer gezeigt hat, $\left.{ }^{1}\right)^{2}$ ) das auf dem Wege der Veresterung vortrefflich branchbare Ausgangsprăparate gewonnen werden können und Pregls Methode der Mikroelementaranalyse zur Verfügung steht, mit der genaue Analysen auch an recht kleinen Mengen von Substanz ausführbar sind, ist lebhaft $z \mathfrak{u}$ wünschen, und wohl nicht $z \mathfrak{u}$ bezweifeln, das auch die Erforschung der chemischen Konstitution der natürlichen Porphyrine in dem einen oder anderen mit den erforderlichen Hilfsmitteln ausgestatteten Institute weiter betrieben werde.

1) H. Fischer, Über das Urinporphyrin, Diese Zeitschrift, Bd. 95, S. 34, 1915.

2) H. Fischer, Über das Kotporphyrin, Diese Zeitschrift, Bd. 96, S. $148,1915$. 